\title{
Using Direct Metal Deposition to Fabricate Mold Plates for an Injection Mold Machine Allowing for the Evaluation of Cost Effective Near-Sourcing Opportunities in Larger, High Volume Consumer Products ${ }^{1}$
}

Radio Systems Corporation

Project ID:

MDF-TC-2013-026

Start Date:

$7 / 25 / 2013$

Completion Date:

$10 / 31 / 2014$

Company Size:

Large business

Report Number:

ORNL/TM-2015/272: Approved for Public Release

\section{Summary}

ORNL collaborated with Radio Systems Corporation to investigate additive manufacturing (AM) of mold plates for plastic injection molding by direct metal deposition. The team's modelling effort identified a 100\% improvement in heat transfer through use of conformal cooling lines that could be built into the mold using a revolutionary design enabled by additive manufacturing. Using the newly installed laser deposition system at the ORNL Manufacturing Demonstration Facility (MDF) a stainless steel mold core was printed.

\section{Background}

The goal of this collaborative project with Radio Systems Corporation was to evaluate the use of Direct Metal Deposition techniques to fabricate mold plates and inserts for injection molding. These mold inserts fit into larger mold bases for use in standard plastic injection mold machines. The capability of additive manufacturing to place heating and cooling lines closer to the actual molding surface (i.e. conformal cooling lines) has the potential of decreasing the cycle time for injection molding process. As these conformally-cooled inserts are used to produce tens of thousands of plastic injection molded parts, a reduction in cycle time of a few seconds per part could dramatically increase the manufacturing speed and improve the business case. This could result in adding manufacturing jobs to the local economy and reducing transportation energy costs significantly. As it currently stands, $90 \%$ of the injection molding plates are manufactured in China and shipped to the US, because domestic manufacturing costs are 3-4 times higher.

\footnotetext{
${ }^{1}$ Research sponsored by the U.S. Department of Energy, Office of Energy Efficiency and Renewable Energy, Advanced Manufacturing Office, under contract DE-AC05-00OR22725 with UT-Battelle, LLC.
} 
The initial goal of this project was to make an injection molding insert for the production of a large cat door for PetSafe. The mechanical criteria for a suitable insert included a minimum material hardness of 2 tons/in $(\mathrm{H}-13$ tool steel) and the ability to maintain a geometric tolerance of $\pm 0.2 \mathrm{~mm}$ without warping up to $300^{\circ} \mathrm{C}$. The Arcam electron beam melting technology was initially targeted for this project, but the mechanical and thermal properties of titanium combined with the cost of the raw stock powder made the business case unfavorable for this particular geometry.

\section{Technical Results}

The first step in the project was then to select a promising candidate geometry for the test case. The product selected was the Aqua Cube Pet Fountain $(60,000$ units/yr, see picture below) - because the base of the product offered both deep and fine geometric features that would likely most benefit from the use of conformal cooling lines. The injection molding process for the Aqua Cube part required pressures of 8-10 ksi and injection temperatures of $450 \mathrm{~F}$.
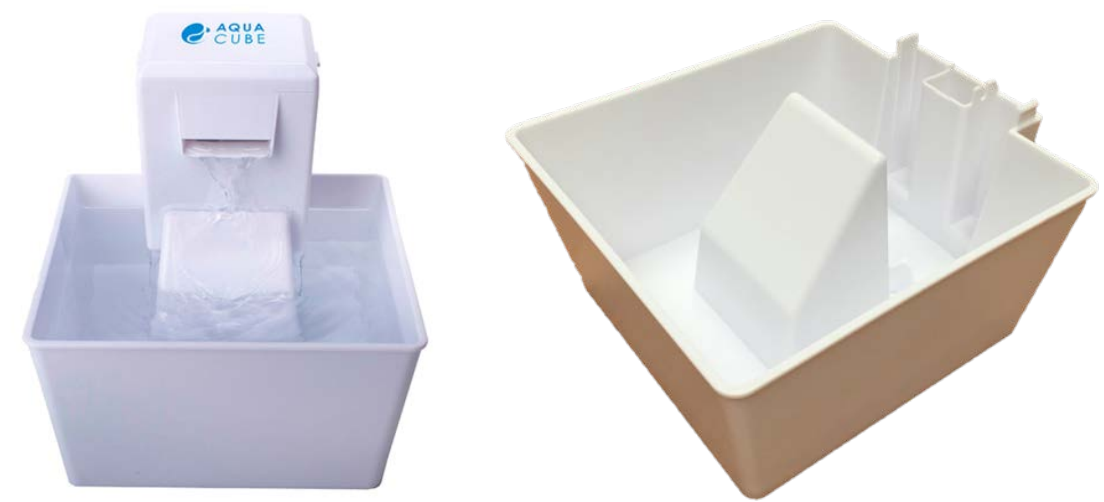

Figure 1. Aqua Cube Pet Fountain (assembled on left, stand-alone base on right)

A transient heat transfer model of the mold insert was developed to compare the effective heat transfer rate for designs involving conformal cooling lines versus conventional cooling lines. A key understanding to come out of the modeling analysis was that the primary impact on heat transfer occurs on the "core" surface of the mold (as opposed to the "cavity" side) because the plastic would shrink around the core during the solidification and cooling process - thus maintaining intimate contact and enabling effective heat transfer. The model also demonstrated that heat flow between core and cavity is minimal and that the thermal bulk of the mold structure dominates cooling efficiency. Modeling also showed that conventional molds have significant temperature variations and are much slower to cool after injection, which limits cycle time. The model showed that the use of conformal cooling lines reduces temperature variation significantly and increases heat transfer at the interface by $100 \%$. It was anticipated that this increase in heat transfer rate could reduce the cycle time of injection molding a part by several seconds, thus improving the throughput on the injection molding machine considerably. 


\section{Conventional Cooling}

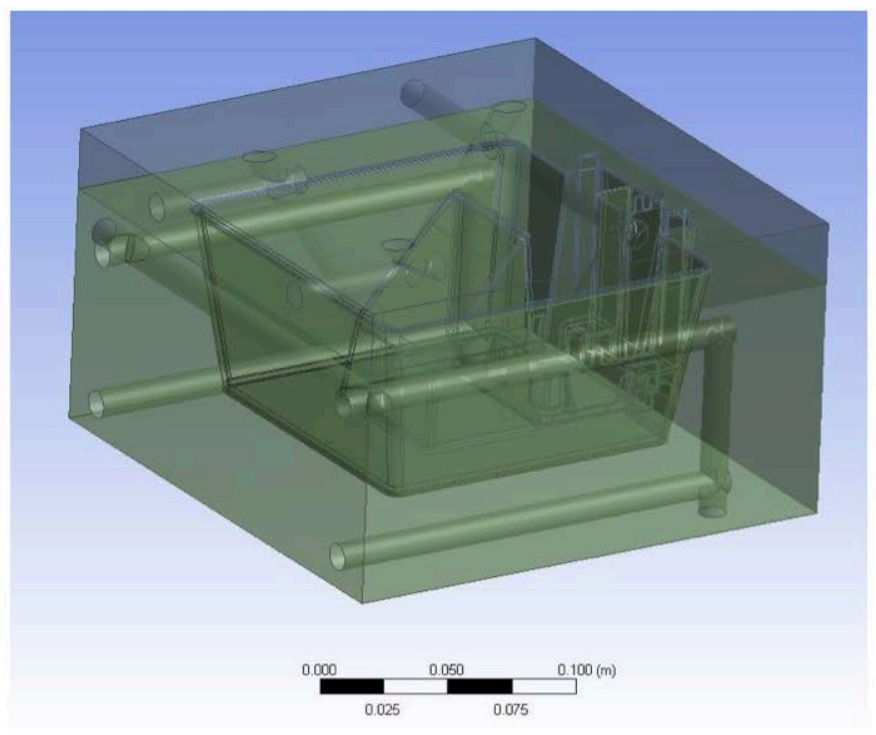

\section{Conformal Cooling}

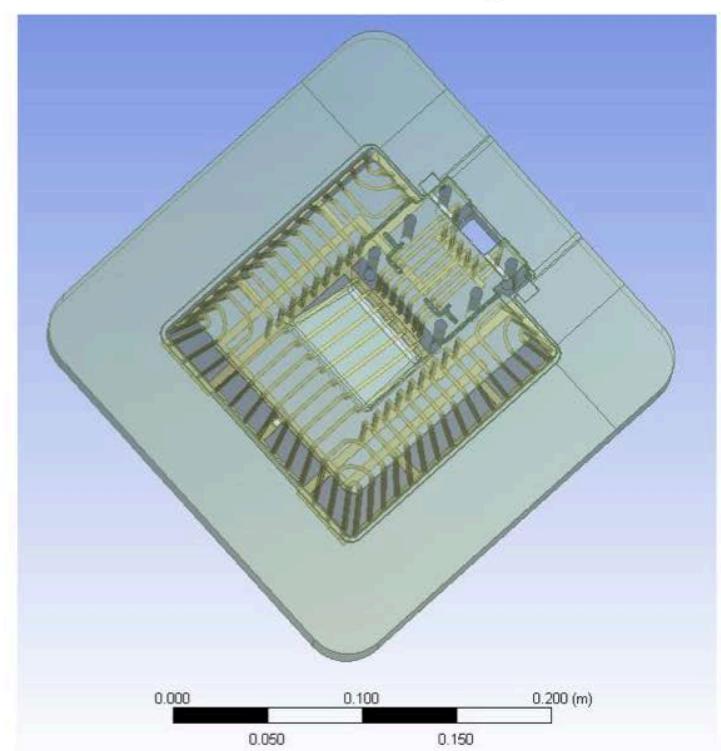

Figure 2. Conventional cooling line layout in the mold core (left) compared to conformal cooling lines (right)

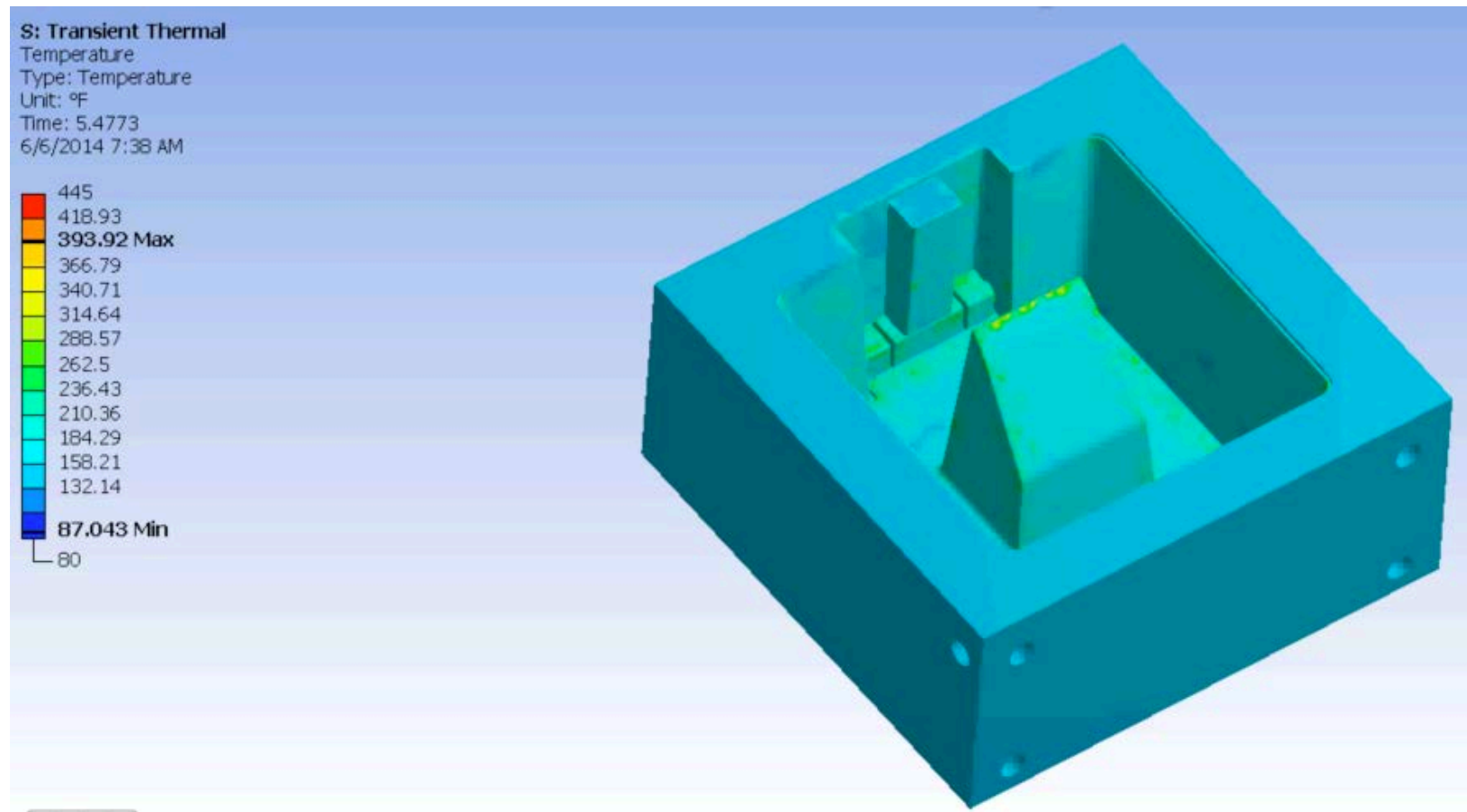

Figure 3. Temperature distribution across conventionally cooled insert 5.5 seconds after injection of molten plastic. 


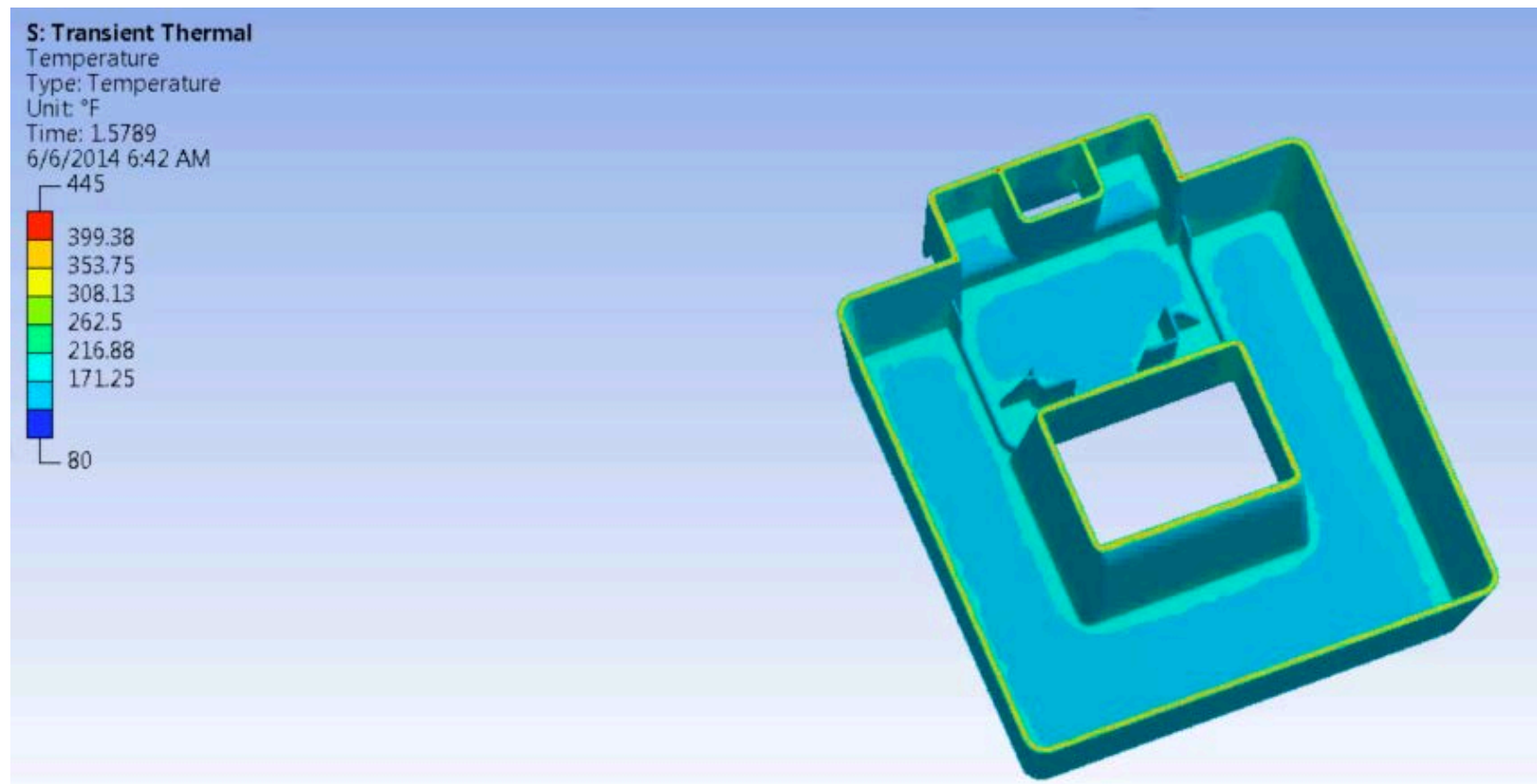

Figure 4. Thermal variations within the injection molded part 1.5 seconds after injection (conventional cooling).

Several cooling line configurations were considered to improve heat transfer as well as improve manufacturability of the part. A "cascading waterfall" approach was thought to have better heat transfer, but would potentially weaken the structural integrity of the mold core too much. An alternative "channeling" approach was considered a good balance between structural integrity and heat transfer (Figure 5).

\section{SECTION VIEWS}

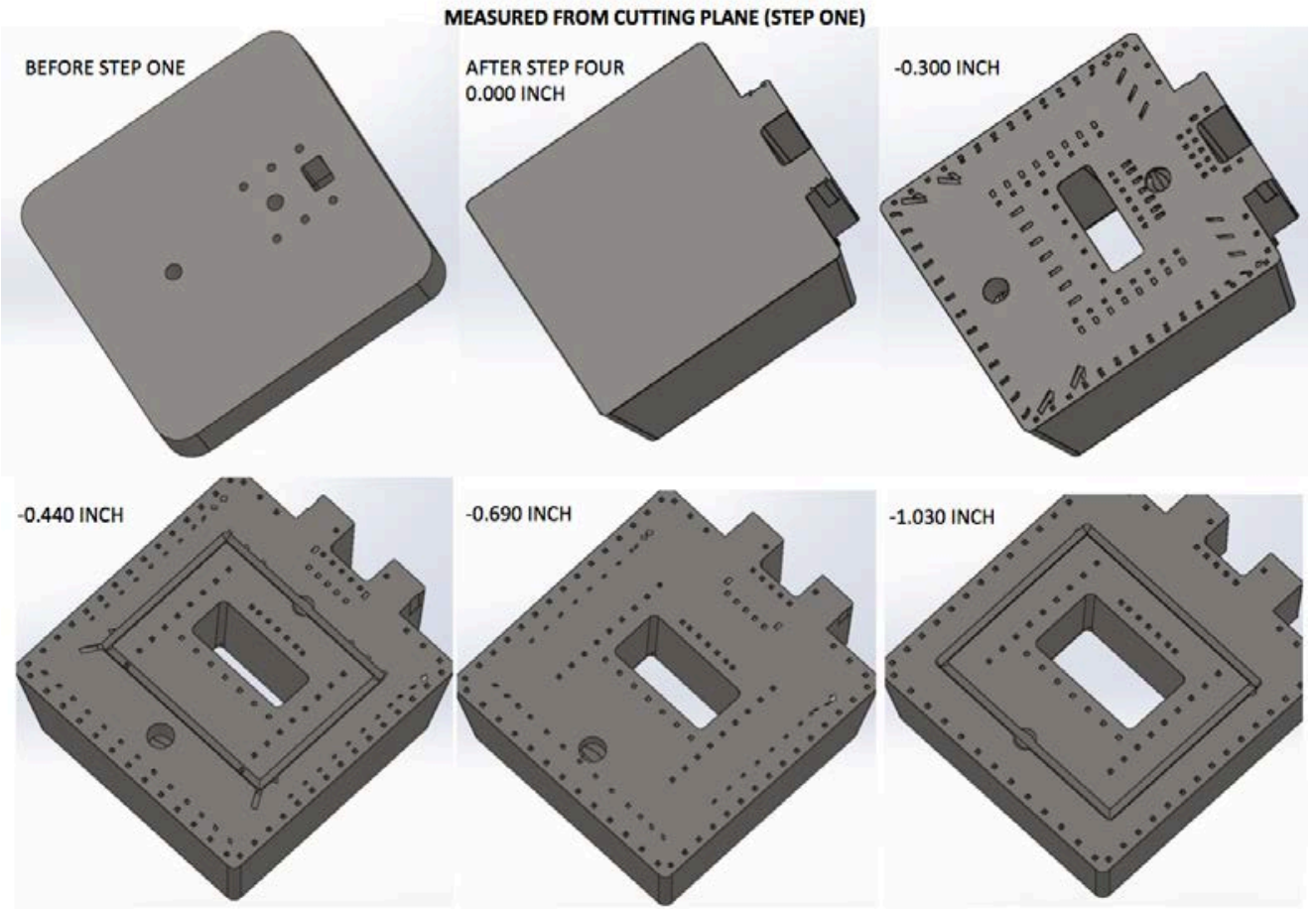

Figure 5. Section views of conformal cooling configuration mold core design. 
The design shown in Figure 5 was manufactured by the MDF as a demonstration mold core using the Renishaw AM250 laser melting machine from 316 Stainless Steel (Figure 6). An innovative approach was used for the manufacture of the mold insert since it was so large - by incorporating the build plate into the design of the mold insert, the manufacturing time was reduced considerably (to $\sim 130$ hours). Unfortunately, the air filter currently used by the Renishaw machine is only rated for $\sim 30$ hours of operation. During the latter portions of the build, it is thought that the lens became dirty and the effective laser power delivered to the build bed was reduced considerably. This caused the metal powder to not completely consolidate and resulted in significant porosity in the finished part. Initial testing of the component at a local injection molding facility (Mountain Mold \& Die) showed the mold insert to be too porous to be used for testing in an injection molding machine.

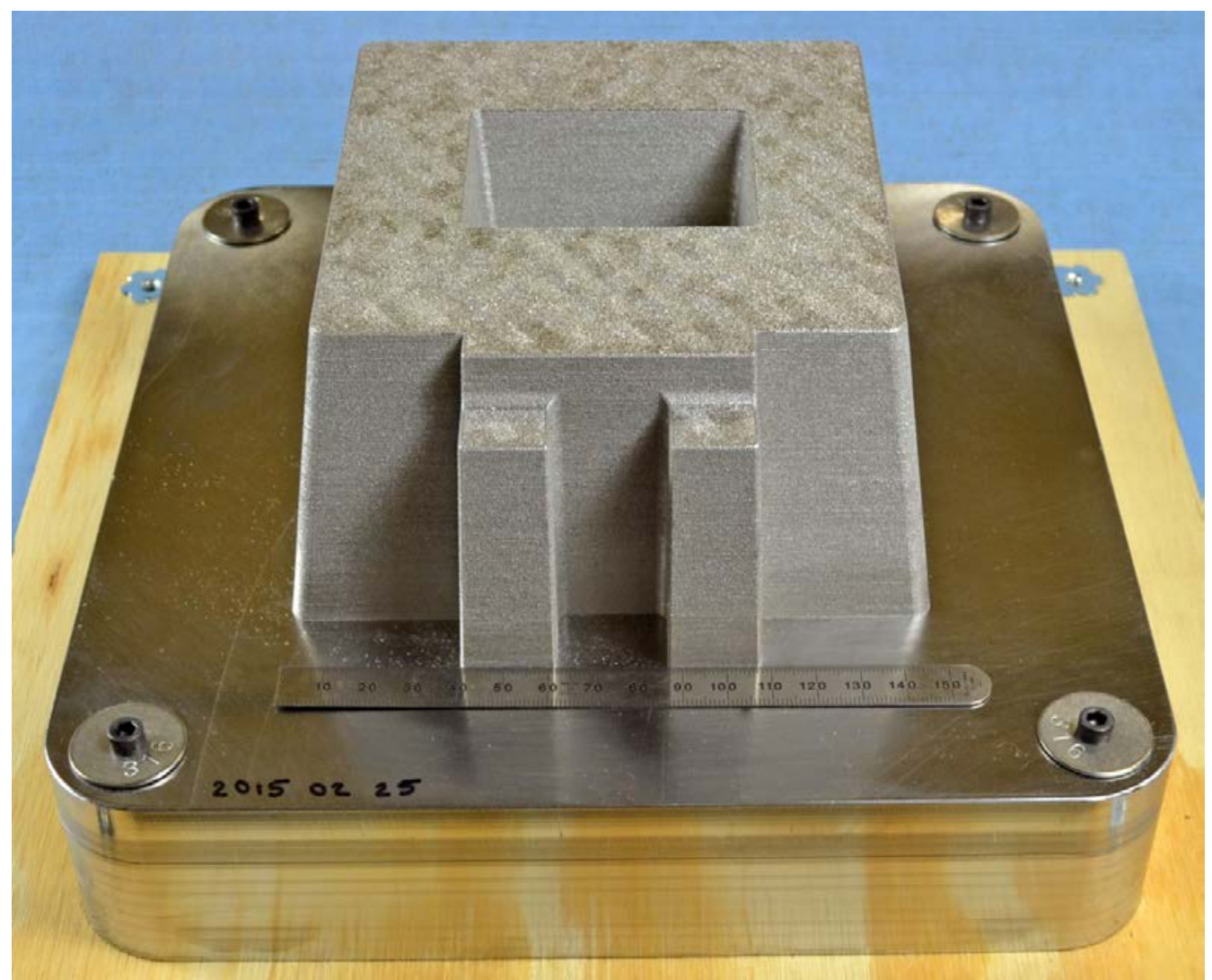

Figure 6. Mold insert manufactured at ORNL's MDF out of 316 stainless steel

\section{Impacts}

This collaborative project between Radio Systems Corporation and the ORNL MDF evaluated the opportunity to use additive manufacturing techniques to fabricate mold inserts for injection molding. This project demonstrated that conformal cooling can dramatically increase the heat transfer rate at the mold interface (by 100\%) which can potentially reduce the cycle time for injection molding, thus improving throughput and productivity for injection molding. The project also demonstrated an innovative approach to the manufacture of a mold insert combined with the build plate of the AM machine, which reduces the build time for the combined component by a factor of $\sim 3 x$. 


\section{Conclusions}

This project demonstrated that conformal cooling designs enabled by additive manufacturing can effectively improve the heat transfer for injection molding inserts and potentially reduce cycle time and improve throughput for injection molded components. A mold insert was manufactured with conformal cooling lines, but was considered too porous for use in injection molding trials. Future efforts need to focus on producing a fully consolidated component for functional testing.

\section{About the Company}

Radio Systems ${ }^{\circledR}$ Corporation (RSC) is the leading manufacturer of pet products. Our family of brands includes PetSafe ${ }^{\circledR}$ (www.petsafe.net), Invisible Fence ${ }^{\circledR}$ Brand (www.invisiblefence.com) and SportDOG ${ }^{\mathrm{TM}}$ (www.sportdog.com). We are a company driven by innovation and dedication to our consumers and communities. Since our founding in 1991, Radio Systems has grown into an international corporation selling in over 52 countries.

\section{Points of Contact}

Chad Duty, ORNL, dutyc@ornl.gov, 865-574-5059

Bill Groh, Radio Systems Corporation, bgroh@petsafe.net, 865-824-5447 